\title{
COMPARISON BETWEEN TEICHMÜLLER METRIC AND LENGTH SPECTRUM METRIC UNDER PARTIAL TWISTS
}

\author{
Jun $\mathrm{Hu}^{*}$ and Francisco G. Jimenez-Lopez ${ }^{\dagger}$
}

Brooklyn College of CUNY, Department of Mathematics, Brooklyn, NY 11210, U.S.A. and Graduate Center of CUNY, Ph.D. Program in Mathematics

365 Fifth Avenue, New York, NY 10016, U.S.A.; junhu@brooklyn.cuny.edu, JHu1@gc.cuny.edu

Cinvestav-IPN, Department of Mathematics

3 Libramiento Norponiente 2000, Real De Juriquilla, 76230 Santiago de Queretaro

Queretaro, Mexico; jimenez@math.cinvestav.edu.mx

\begin{abstract}
Let $d_{L}$ and $d_{T}$ denote, respectively, the length spectrum metric and the Teichmüller metric on the Teichmüller space $T\left(S_{0}\right)$ of a Riemann surface $S_{0}$. Wolpert showed that $d_{L}\left(\tau, \tau^{*}\right) \leq$ $d_{T}\left(\tau, \tau^{*}\right)$ for any two points $\tau$ and $\tau^{*}$ in $T\left(S_{0}\right)$. If $S_{0}$ is a hyperbolic Riemann surface with nonelementary Fuchsian group, then there are two sequences $\left\{\tau_{n}\right\}$ and $\left\{\tau_{n}^{*}\right\}$ of points in $T\left(S_{0}\right)$ such that $d_{L}\left(\tau_{n}, \tau_{n}^{*}\right) \rightarrow 0$ as $n \rightarrow \infty$, but $d_{T}\left(\tau_{n}, \tau_{n}^{*}\right) \geq b$ for some positive constant $b$ and any $n$. This property was proved in [11] for any hyperbolic compact Riemann surface and in [13] for any hyperbolic one with non-elementary Fuchsian group. It is further shown in [13] that the two sequences can be modified to keep $d_{L}\left(\tau_{n}, \tau_{n}^{*}\right) \rightarrow 0$ but have $d_{T}\left(\tau_{n}, \tau_{n}^{*}\right) \rightarrow \infty$ as $n \rightarrow \infty$. For all these results, each $\tau_{n}^{*}$ is constructed from a Riemann surface $\tau_{n}$ by taking a number of Dehn twists (full twists) along a closed curve on $\tau_{n}$. When $\tau_{n}^{*}$ is constructed from $\tau_{n}$ through a Dehn twist, one can use the maximal dilatation of a quasiconformal self map of $\tau_{n}$ to control and compare $d_{L}\left(\tau_{n}, \tau_{n}^{*}\right)$ and $d_{T}\left(\tau_{n}, \tau_{n}^{*}\right)$. But when $\tau_{n}^{*}$ is constructed from $\tau_{n}$ in a similar pattern but with partial twist, the method of using a self map of $\tau_{n}$ to control and compare $d_{L}\left(\tau_{n}, \tau_{n}^{*}\right)$ and $d_{T}\left(\tau_{n}, \tau_{n}^{*}\right)$ fails. In this paper, we show how to control and compare $d_{L}\left(\tau, \tau^{*}\right)$ and $d_{T}\left(\tau, \tau^{*}\right)$ under such partial twists, which enables us to obtain continuous versions of the results of [11] and [13] by using partial twists to connect the points $\tau_{n}^{*}$.
\end{abstract}

\section{Introduction}

Let $S_{0}$ be a Riemann surface. A marked Riemann surface is a pair $(S, f)$ with $f: S_{0} \rightarrow S$ being a quasiconformal mapping. Two pairs $\left(S_{1}, f_{1}\right)$ and $\left(S_{2}, f_{2}\right)$ are equivalent if there exists a conformal mapping $c: S_{1} \rightarrow S_{2}$ such that $c \circ f_{1}$ is homotopic to $f_{2}$. The Teichmüller space $T\left(S_{0}\right)$ is the set of equivalence classes $[S, f]$.

The Teichmüller metric on $T\left(S_{0}\right)$ is defined by

$$
d_{T}\left(\left[S_{1}, f_{1}\right],\left[S_{2}, f_{2}\right]\right)=\inf _{f} \log K(f)
$$

where $f$ ranges over all quasiconformal mappings between $S_{1}$ and $S_{2}$ homotopic to $f_{2} \circ f_{1}^{-1}$ and $K(f)$ represents the maximal dilatation of $f$.

We consider another metric on $T\left(S_{0}\right)$, called the length spectrum metric. Let $S$ be a Riemann surface of the same type as $S_{0}$. A simple closed curve on $S$ is said to be essential if it is neither homotopic to a point nor to a puncture and nor to a boundary

doi:10.5186/aasfm.2016.4121

2010 Mathematics Subject Classification: Primary 30F60.

Key words: Teichmüller metric, length spectrum metric, partial twist, earthquake map.

* Research partially supported by PSC-CUNY research awards.

${ }^{\dagger}$ Research partially supported by CONACYT posdoctoral fellowship. 
component. Let $\Sigma_{S}$ be the collection of simple closed curves on $S$ containing one and exactly one representative from each homotopy class of essential curves. For each $\gamma \in \Sigma_{S}$, let $l_{S}(\gamma)$ denote the length of the shortest curve in the homotopy class of $\gamma$ in the hyperbolic metric. The length spectrum metric is defined by

$$
d_{L}\left(\left[S_{1}, f_{1}\right],\left[S_{2}, f_{2}\right]\right)=\log \sup _{\gamma \in \Sigma_{S_{1}}}\left\{\frac{l_{S_{2}}\left(f_{2} \circ f_{1}^{-1}(\gamma)\right)}{l_{S_{1}}(\gamma)}, \frac{l_{S_{1}}(\gamma)}{l_{S_{2}}\left(f_{2} \circ f_{1}^{-1}(\gamma)\right)}\right\} .
$$

This metric was introduced and studied by Sorvali [17] in 1972. In 1975, Sorvali [18] proved that the Teichmüller metric $d_{T}$ and the length spectrum metric $d_{L}$ are metrically equivalent on the Teichmüller space of a torus and posed a question as to whether or not this is true in general. A related question is whether or not these two metrics induce the same topology on Teichmüller space.

A well known result by Wolpert (see [1]) states that if $f: S_{1} \rightarrow S_{2}$ is a quasiconformal mapping, then

$$
\frac{l_{S_{2}}(f(\gamma))}{l_{S_{1}}(\gamma)} \leq K(f)
$$

for all $\gamma \in \Sigma_{S_{1}}$. This inequality implies that

$$
d_{L}\left(\tau_{1}, \tau_{2}\right) \leq d_{T}\left(\tau_{1}, \tau_{2}\right) \text { for any } \tau_{1}, \tau_{2} \in T\left(S_{0}\right)
$$

Thus Sorvali's question is to study whether or not there exists a positive constant $C$ such that

$$
d_{T}\left(\tau_{1}, \tau_{2}\right) \leq C d_{L}\left(\tau_{1}, \tau_{2}\right) \text { for any } \tau_{1}, \tau_{2} \in T\left(S_{0}\right),
$$

or even weaker, whether or not the identity map

$$
\mathrm{id}:\left(T\left(S_{0}\right), d_{L}\right) \rightarrow\left(T\left(S_{0}\right), d_{T}\right)
$$

is continuous.

In 1986, Li [10] showed that the identity map is continuous if $S_{0}$ is a compact Riemann surface. This result was later generalized by Liu in [12] to the case where $S_{0}$ is a Riemann surface of type $(g, m, n)$, where $g, m$ and $n$ are the genus, number of punctures and number of ideal boundaries, respectively, with $6 g-6+m+3 n>0$. Then it follows that these two metrics are topologically equivalent on the Teichmüller space $T\left(S_{0}\right)$. Furthermore, in $2003 \mathrm{Li}$ [11] proved that these two metrics are not metrically equivalent on the Teichmüller space of a compact Riemann surface of genus $g \geq 2$. In order to prove this, he first constructed a sequence $\left\{\tau_{n}=\left[S_{n}, f_{n}\right]\right\}$ of points in $T\left(S_{0}\right)$ such that $S_{n}$ contains a closed curve $\beta_{n}$ with $\lim _{n \rightarrow \infty} l_{S_{n}}\left(\beta_{n}\right)=0$. Then, by taking $\left[1 / l_{S_{n}}\left(\beta_{n}\right)\right]$ number of Dehn twists along each $\beta_{n}$, he obtained another sequence $\left\{\tau_{n}^{*}\right\}$ of points in $T\left(S_{0}\right)$ such that $d_{T}\left(\tau_{n}, \tau_{n}^{*}\right) \geq d>0$ for $n$ sufficiently large. On the other hand, since $l_{S_{n}}\left(\beta_{n}\right) \rightarrow 0$, it follows from the Collar Lemma [7] that the effect of that number of Dehn twists on the hyperbolic length of any curve crossing $\beta_{n}$ becomes smaller and smaller as $n$ approaching $\infty$. This means $d_{L}\left(\tau_{n}, \tau_{n}^{*}\right) \rightarrow 0$ as $n \rightarrow \infty$.

Liu, Sun and Wei [13] generalized and improved Li's result to the Teichmüller space of any hyperbolic Riemann surface with non-elementary Fuchsian group by finding two sequences $\left\{\tau_{n}\right\}$ and $\left\{\tau_{n}^{*}\right\}$ such that $d_{T}\left(\tau_{n}, \tau_{n}^{*}\right) \rightarrow \infty$ but $d_{L}\left(\tau_{n}, \tau_{n}^{*}\right) \rightarrow 0$ as $n \rightarrow \infty$. Their construction follows Li's idea but uses more Dehn twists to obtain the sequence $\left\{\tau_{n}^{*}\right\}$. In their constructions, each $\tau_{n}^{*}$ can be represented by a quasiconformal mapping from $S_{n}$ onto itself, which comes from cutting $S_{n}$ along $\beta_{n}$ and then gluing the two copies of $\beta_{n}$ back after twisting one of the two copies three hundred and sixty degrees enough times. This feature makes it feasible to estimate the length 
spectrum distance between $\tau_{n}$ and $\tau_{n}^{*}$ by using ratios of the lengths of closed curves and their images under a homeomorphism defined on the same Riemann surface. This convenience of representing $\tau_{n}^{*}$ is lost when a partial twist is performed to construct a new point in $T\left(S_{0}\right)$. In this case, the new point has to be represented by maps between two Riemann surfaces with different hyperbolic metrics. In this paper, we first show how to control the length spectrum distance between two points $\tau$ and $\tau^{*}$ in $T\left(S_{0}\right)$ when $\tau^{*}$ is constructed through a partial twist along a closed geodesic $\beta$ on $\tau$. The points $\tau_{n}$ are connected by a nice curve in $T\left(S_{0}\right)$. Now we use continuously changed partial twists along closed geodesics on the points on the nice curve to obtain another continuous curve in $T\left(S_{0}\right)$ to connect the points $\tau_{n}^{*}$ and in the meantime we have the properties of $\tau_{n}$ and $\tau_{n}^{*}$ in [11] and [13] preserved for the points on the two curves. More precisely, we prove the following theorem.

Theorem 1. Let $S_{0}$ be a hyperbolic Riemann surface with non-elementary Fuchsian group. Then there exist three curves $\alpha(t), \alpha^{*}(t)$ and $\hat{\alpha}(t), 0 \leq t<1$, in $T\left(S_{0}\right)$ having the following properties.

(1) $\lim _{t \rightarrow 1} d_{T}\left(\alpha(t), \alpha^{*}(t)\right)=\infty$, but $\lim _{t \rightarrow 1} d_{L}\left(\alpha(t), \alpha^{*}(t)\right)=0$.

(2) There exist $M, m>0$ such that $m \leq d_{T}(\alpha(t), \hat{\alpha}(t)) \leq M$ for all $t$, but $\lim _{t \rightarrow 1} d_{L}(\alpha(t), \hat{\alpha}(t))=0$.

Moreover, the curve $\alpha$ can be chosen to be a Teichmüller geodesic ray.

Our theorem can be viewed as continuous versions of the main results of [11] and [13].

Remark 1. In 2003, Shiga [16] studied the relation between the length spectrum metrc and the Teichmüller metric on Riemann surfaces $S_{0}$ of infinite type. He showed that if $S_{0}$ has a sequence of closed geodesics with lengths approaching 0 , then one of the sequences in the result of Liu, Sun and Wei [13] can be reduced to a point. That is, in the Teichmüller space $T\left(S_{0}\right)$ of such a Riemann surface $S_{0}$ there is a sequence of points $\left\{\tau_{n}\right\}_{n=0}^{\infty}$ such that $d_{L}\left(\tau_{n}, \tau_{0}\right) \rightarrow 0$ but $d_{T}\left(\tau_{n}, \tau_{0}\right) \rightarrow \infty$ as $n \rightarrow \infty$, where $\tau_{0}$ is the base point of $T\left(S_{0}\right)$. This result implies that the topologies defined by the two metrics are not equivalent. It also raises a problem on how to connect the points $\left\{\tau_{n}\right\}_{n=1}^{\infty}$ by a continuous curve in $T\left(S_{0}\right)$ such that Shiga's result continues to hold on the curve. Since Shiga's construction of the points $\tau_{n}, n \geq 1$, involves with different amounts of Dehn twists around infinitely many different closed geodesics, the technique developed in this paper doesn't seem enough to solve the problem.

Acknowledgement. Both authors wish to thank the referees for their comments, suggestions and corrections of typos. Especially, they are grateful to one of them for the suggestion to generalize our result from a hyperbolic Riemann surface of finite topological type to any hyperbolic Riemann surface with non-elementary Fuchsian group, which leads to the current version of the paper. They also wish to thank Professors Frederick Gardiner and Linda Keen for inspiring discussions.

\section{Earthquakes}

Earthquakes were introduced by Thurston in [21] to measure the difference between two conformal structures on a surface or two points in the Teichmüller space of a Riemann surface. For background on earthquake maps and their relations with quasisymmetric circle homeomorphisms, we refer to $[3,4,5,15]$. 
A lamination $\mathcal{L}$ on $\mathbf{D}$ is a collection of hyperbolic geodesics that foliates a closed subset of $\mathbf{D}$. The geodesics in $\mathcal{L}$ are called leaves, whereas the connected components of $\mathbf{D} \backslash \mathcal{L}$ are called gaps. The strata of the lamination $\mathcal{L}$ consists of gaps and leaves. A generalized left earthquake map $\widetilde{E}: \mathbf{D} \rightarrow \mathbf{D}$ supported on $\mathcal{L}$ is a possibly discontinuous injective map which is an isometry on each stratum of $\mathcal{L}$. Even more, for any two strata $A \neq B$, the comparison isometry

$$
\operatorname{Comp}(A, B)=\left(\left.\widetilde{E}\right|_{A}\right)^{-1} \circ\left(\left.\widetilde{E}\right|_{B}\right)
$$

is a hyperbolic translation whose axis weakly separates $A$ and $B$ and which translates $B$ to the left as viewed from $A$.

If in addition the map $\widetilde{E}$ is surjective, we call it a left earthquake map.

Thurston [21] showed that each left earthquake map $\widetilde{E}: \mathbf{D} \rightarrow \mathbf{D}$ extends to a map defined on $\mathbf{D} \cup \mathbf{S}^{1}$. The extension is continuous at each point of $\mathbf{S}^{1}$ and $\left.\widetilde{E}\right|_{\mathbf{S}^{1}}$ is a homeomorphism. Conversely, every circle homeomorphism can be realized in this way.

Theorem 2. (Thurston) Let $h$ be an orientation-preserving homeomorphism of the unit circle $\mathbf{S}^{1}$. Then there exists a lamination $\mathcal{L}$ and a left earthquake map $\widetilde{E}: \mathbf{D} \rightarrow \mathbf{D}$ along the leaves in $\mathcal{L}$ such that $\left.\widetilde{E}\right|_{\mathbf{S}^{1}}=h$. The lamination is uniquely determined by $h$. Moreover, $h$ determines the isometries of $\widetilde{E}$ on all gaps, and for any leaf $L$ in $\mathcal{L}$, two possibly different isometries on $L$ only differ by a hyperbolic isometry with axis $L$ and translation length between 0 and the limit value of the translation lengths of the comparison maps for $E$ on the two sides of $L$.

Each generalized earthquake map $\widetilde{E}: \mathbf{D} \rightarrow \mathbf{D}$ along the leaves in $\mathcal{L}$ induces a transverse measure $\sigma$, called an earthquake measure on $\mathcal{L}$. The measure $\sigma$ quantifies the amount of relative shearing along the lamination of the earthquake map.

The following result is due to Thurston [21]. A proof is given in [3].

Theorem 3. (Thurston) Let $\sigma$ be a transverse measure defined on a lamination $\mathcal{L}$. Then there exists a generalized earthquake map $\widetilde{E}: \mathbf{D} \rightarrow \mathbf{D}$ supported on $\mathcal{L}$ such that $\sigma$ is the induced earthquake measure by $\widetilde{E}$. Moreover, up to post-composition by a hyperbolic isometry, $\sigma$ determines the isometries of $\widetilde{E}$ on all gaps, and for any leaf $L$ in $\mathcal{L}$, two possible isometries on $L$ only differ by a hyperbolic isometry with axis $L$ and translation length between 0 and the measure $\sigma(L)$ of $L$.

In order to determine whether or not a generalized earthquake map is indeed an earthquake map we need to introduce the concept of the Thurston norm of an earthquake measure $(\sigma, \mathcal{L})$.

Let $\mathcal{L}$ be a lamination with a transverse measure $\sigma$. The Thurston norm of $\sigma$ is defined to be

$$
\|\sigma\|_{\mathrm{Th}}=\sup \sigma(\alpha)
$$

where the supremum is taken over all closed geodesic segments $\alpha$ of length 1 that are transverse to the lamination.

We say that $\sigma$ is Thurston bounded if $\|\sigma\|_{\text {Th }}$ is finite. An earthquake map is called Thurston bounded if the induced earthquake measure is Thurston bounded.

Thurston [21] outlined the proofs of the following two theorems. Three different complete proofs are given in $[3,4,15]$.

Theorem 4. (Thurston) Let $\mathcal{L}$ be a lamination with a transverse measure $\sigma$. If $\sigma$ is Thurston bounded, then there exists a left earthquake map $\widetilde{E}: \mathbf{D} \rightarrow \mathbf{D}$ supported 
on $\mathcal{L}$ such that $\sigma$ is the induced earthquake measure by $\widetilde{E}$. Moreover, up to postcomposition by a hyperbolic isometry, $\sigma$ determines the isometries of $\widetilde{E}$ on all gaps, and for any leaf $L$ in $\mathcal{L}$, two possible isometries on $L$ only differ by a hyperbolic isometry with axis $L$ and translation length between 0 and the measure $\sigma(L)$ of $L$.

Theorem 5. (Thurston) Let $h$ be an orientation-preserving circle homeomorphism, and let $\sigma_{h}$ be the earthquake measure induced by a left earthquake map $\widetilde{E}$ with $\left.\widetilde{E}\right|_{\mathbf{S}^{1}}=h$. Then $\sigma_{h}$ is Thurston bounded if and only if $h$ is quasisymmetric.

For situations handled in this paper, we are only interested in discrete laminations, i.e., laminations consisting of countably many hyperbolic geodesics without any accumulation in $\mathbf{D}$. From now on, we assume that this is the case. We now construct generalized earthquakes for these types of laminations with transverse measures.

Let $\mathcal{L}$ be a discrete lamination. Two gaps are called neighbors if there is a geodesic in $\mathcal{L}$, called the separating geodesic, that belongs to the boundary of both gaps. Given any two gaps $A$ and $B$, there exists a unique minimal chain of gaps $A_{0}=A, A_{1}, A_{2}, \ldots, A_{n+1}=B$ such that $A_{i}$ and $A_{i+1}$ are neighbors. In this sense, the pattern of neighboring gaps determined by $\mathcal{L}$ is a tree.

Let $\sigma$ be a measure on a lamination $\mathcal{L}$. That is, $\sigma$ assigns a positive number, called a weight, to each element of $\mathcal{L}$. Fix a gap $A$ of the lamination $\mathcal{L}$ and define $\left.\widetilde{E}\right|_{A}=$ Id. Let $B$ be any other gap and let $A_{0}=A, A_{1}, \ldots, A_{n+1}=B$ be the minimal chain of neighboring gaps between $A$ and $B$. For each $i=1,2, \ldots, n+1$, let $T_{i}$ be the hyperbolic translation whose axis is the separating geodesic $L_{i}$ between $A_{i-1}$ and $A_{i}$ and that translates $A_{i}$ to the left by a distance $\lambda_{i}$, where $\lambda_{i}$ is the weight of $L_{i}$. Define $\left.\widetilde{E}\right|_{B}=T_{1} \circ T_{2} \circ \cdots \circ T_{n+1}$. Since any two gaps are connected by a unique minimal chain of neighboring gaps, this construction gives a map defined on the whole hyperbolic plane. Of course, this construction depends on the gap $A$, nonetheless, any two maps constructed in this way differ only by pre-composition by a conformal isometry of $\mathbf{D}$. It is easy to see that this map is injective and that the comparison isometry satisfies condition (2.1), however, $\widetilde{E}$ might not be surjective.

A Thurston bounded earthquake map is a quasi-isometry on $\mathbf{D}$ with respect to the hyperbolic metric. The Thurston norm of the earthquake measure is a quantifier of the quasi-isometry. On the other hand, the cross-ratio distortion norm is a quantifier of the quasisymmetry of the boundary homeomorphism determined by the earthquake map. Now we introduce the definition of the cross-ratio distortion norm and we give its quantitative relationship with the Thurston norm of the measure.

Let $h$ be an orientation-preserving homeomorphism of the unit circle $\mathbf{S}^{1}$. The cross-ratio distortion norm $\|h\|_{\text {cr }}$ of $h$ is defined as

$$
\|h\|_{\mathrm{cr}}=\sup _{Q}\left|\log \frac{\operatorname{cr}(h(Q))}{\operatorname{cr}(Q)}\right| \text {, }
$$

where the supremum is taken over all quadruples $Q=\{a, b, c, d\}$ of four points arranged in counter-clockwise order on the unit circle with $\operatorname{cr}(Q)=1$, and where

$$
\operatorname{cr}(Q)=\frac{(b-a)(d-c)}{(c-b)(d-a)} .
$$

Theorem 6. (Gardiner-Hu-Lakic) There exists a universal constant $C^{\prime}>0$ such that for any measured lamination $(\mathcal{L}, \sigma)$,

$$
\frac{1}{C^{\prime}}\left\|\left.\widetilde{E}\right|_{\mathbf{S}^{1}}\right\|_{\mathrm{cr}} \leq\|\sigma\|_{\mathrm{Th}} \leq C^{\prime}\left\|\left.\widetilde{E}\right|_{\mathbf{S}^{1}}\right\|_{\mathrm{cr}}
$$


The right inequality in the previous theorem was proved by Gardiner, $\mathrm{Hu}$ and Lakic in [3] and the left inequality was proved by $\mathrm{Hu}$ in [4].

For any orientation-preserving homeomorphism $f$ of the unit circle $\mathbf{S}^{1}$, let $\Phi(f)$ be the Douady-Earle extension of $f$ (see [2]) and let $K_{e}(f)$ be the infimum of maximal dilatations of quasiconformal extensions of $f$. Hu and Muzician [6] proved the following two theorems.

Theorem 7. (Hu-Muzician) There exists a universal constant $C^{\prime \prime}>0$ such that for any quasisymmetric circle homeomorphism $f$,

$$
\log K(\Phi(f)) \leq C^{\prime \prime}\|f\|_{\mathrm{cr}}
$$

Theorem 8. (Hu-Muzician) Let $f$ be a quasisymmetric homeomorphism. Then

$$
\lim _{\|f\|_{\mathrm{cr}} \rightarrow \infty} \frac{\|f\|_{\mathrm{cr}}}{K_{e}(f)} \leq \pi
$$

The following property is also used in the next section.

Proposition 1. Let $\left\{f_{n}\right\}$ be a sequence of orientation-preserving homeomorphisms of the unit circle $\mathbf{S}^{1}$ such that $K_{e}\left(f_{n}\right) \rightarrow 1$ as $n \rightarrow \infty$. Then

$$
\left\|f_{n}\right\|_{\text {cr }} \rightarrow 0 \text { as } n \rightarrow \infty \text {. }
$$

Proof. Suppose that the proposition is not true. By passing to a subsequence, we may assume that there exists $\varepsilon>0$ such that

$$
\left\|f_{n}\right\|_{\mathrm{cr}}>\varepsilon \text { for all } n \text {. }
$$

For each $n$, choose a quadrilateral $Q_{n}$ such that

$$
\operatorname{cr}\left(Q_{n}\right)=1 \quad \text { and } \quad\left|\log \operatorname{cr}\left(f_{n}\left(Q_{n}\right)\right)\right|>\varepsilon .
$$

We may assume, by post-composing and pre-composing by Möbius transformations, that $Q_{n}$ and $f_{n}\left(Q_{n}\right)$ are the quadrilaterals on $\mathbf{H}$ with vertices at $\{-1,0,1, \infty\}$ and $\left\{x_{n}, 0,1, \infty\right\}$ respectively. For each $n$, the conformal modulus $\operatorname{Mod}\left(Q_{n}\right)$ of $Q_{n}$ is equal to 1 and

$$
\frac{\operatorname{Mod}\left(Q_{n}\right)}{K_{e}\left(f_{n}\right)} \leq \operatorname{Mod}\left(f_{n}\left(Q_{n}\right)\right) \leq K_{e}\left(f_{n}\right) \operatorname{Mod}\left(Q_{n}\right)
$$

Using the assumption that $K_{e}\left(f_{n}\right) \rightarrow 1$ as $n \rightarrow \infty$, we conclude that $\operatorname{Mod}\left(f_{n}\left(Q_{n}\right)\right) \rightarrow$ 1 and then $x_{n} \rightarrow-1$ as $n \rightarrow \infty$. Since $\operatorname{cr}\left(f_{n}\left(Q_{n}\right)\right)=-x_{n}$, it follows that $\log \operatorname{cr}\left(f_{n}\right.$ $\left.\left(Q_{n}\right)\right) \rightarrow 0$ as $n \rightarrow \infty$, contradicting (2.8).

Remark 2. Using an elliptic integral depending on a parameter and the work on pp. 59-60 of [9], one can see how $\left\|f_{n}\right\|_{\text {cr }}$ depends on $K_{e}\left(f_{n}\right)$ asymptotically when $K_{e}\left(f_{n}\right) \rightarrow 1$ as $n \rightarrow \infty$, from which the previous proposition follows as well.

\section{Partial twists on Riemann surfaces}

Let $\beta$ be a simple closed geodesic on a hyperbolic Riemann surface $S$ that is not homotopic to a boundary component of $S$. As pointed out in the introduction, the point created by a Dehn twist along $\beta$ in $T(S)$ is described by a quasiconformal map from $S$ onto $S$, which comes from cutting $S$ along $\beta$ and then gluing the two copies of $\beta$ back after twisting one copy of $\beta$ three hundred and sixty degrees. The first goal of this section is to introduce how partial twists (not equal to multiples of $360^{\circ}$ ) along $\beta$ create new points in $T(S)$. This is related to conjugations of the Fuchsian group representing $S$ by earthquake maps corresponding to partial twists. 
Let $\pi: \mathbf{D} \rightarrow S$ be the universal covering map, where $\mathbf{D}$ denotes the unit disk. The preimage $\pi^{-1}(\beta)$ consists of a union of non intersecting geodesics. By assigning the weight $\lambda$ to each one of these geodesics, we obtain a measured lamination $\left(\sigma_{\lambda}, \mathcal{L}\right)$ on $\mathbf{D}$. We can see that the Thurston norm $\left\|\sigma_{\lambda}\right\|_{\mathrm{Th}}$ is finite. Let $D>0$ be the width of a collar neighborhood around $\beta$. Given any geodesic segment $\alpha$ of length 1 transversal to the lamination $\mathcal{L}, \sigma_{\lambda}(\alpha)=\lambda$ if $\alpha$ intersects only one leaf of $\mathcal{L}$; if $\alpha$ intersects $n>1$ leaves of $\mathcal{L}$, then $1=l(\alpha) \geq n D / 2$ and hence $\sigma_{\lambda}(\alpha) \leq \frac{\lambda}{D / 2}$. In summary,

$$
\lambda \leq\left\|\sigma_{\lambda}\right\|_{\mathrm{Th}} \leq \max \left\{\lambda, \frac{\lambda}{D / 2}\right\}
$$

It follows from Theorems 4 and 5 that any left earthquake map $\widetilde{E}: \mathbf{D} \rightarrow \mathbf{D}$ inducing $\left(\sigma_{\lambda}, \mathcal{L}\right)$ is onto and $\left.\widetilde{E}\right|_{\mathbf{S}^{1}}: \mathbf{S}^{1} \rightarrow \mathbf{S}^{1}$ is a quasisymmetric homeomorphism of the unit circle.

Let $G$ be the Fuchsian group uniformizing $S$. For each element $g \in G, \widetilde{E} g \widetilde{E}^{-1}$ is conformal on every gap of the lamination $\mathcal{L}$. Moreover, it is continuous on $\mathbf{D}$. In order to prove this, consider a geodesic $L^{\prime}$ of the lamination $E(\mathcal{L})$. Since $\widetilde{E}^{-1}$ is a right earthquake, along $L^{\prime}$ it splits the hyperbolic plane into two half disks and moves the gap $V^{\prime}$ on one side of $L^{\prime}$ to the right with respect to the gap $U^{\prime}$ on the other side of $L^{\prime}$. Because $g$ is an isometry and it maps a leaf of $\mathcal{L}$ to another leaf of $\mathcal{L}$, it follows that $g$ maps $\widetilde{E}^{-1}\left(V^{\prime}\right)$ and $\widetilde{E}^{-1}\left(U^{\prime}\right)$ to two adjacent gaps $V$ and $U$ of $\mathcal{L}$ separated by $\widetilde{E}^{-1}\left(L^{\prime}\right)$, which is a leaf $L$ of $\mathcal{L}$. In the meantime, $g \circ \widetilde{E}^{-1}$ is also a right earthquake without changing the comparison along $L^{\prime}$. Finally, since $\widetilde{E}$ is a left earthquake and $L \in \mathcal{L}$, it undoes the discontinuity of $g \circ \widetilde{E}^{-1}$ along $L^{\prime}$. Therefore, for every $g \in G$, the mapping $\widetilde{E} g \widetilde{E}^{-1}$ is conformal on $\mathbf{D}$ and hence it defines an isomorphism between $G$ and a new Fuchsian group $G^{\prime}=\widetilde{E} G \widetilde{E}^{-1}$. Let $S^{\prime}=\mathbf{D} / G^{\prime}$. The earthquake $\tilde{E}$ projects to a quasi-isometry

$$
E_{\beta, \lambda}: S \rightarrow S^{\prime}
$$

which is called a left earthquake map of twist or shear $\lambda$ along $\beta$. It is a discontinuous map as soon as $\lambda \neq 0$. In the following, we introduce a quasiconformal map to represent the same point of $E_{\beta, \lambda}: S \rightarrow S^{\prime}$ in the Teichmüller space $T(S)$.

The Douady-Earle extension $\Phi\left(\left.\widetilde{E}\right|_{\mathbf{S}^{1}}\right): \overline{\mathbf{D}} \rightarrow \overline{\mathbf{D}}$ of $\left.\widetilde{E}\right|_{\mathbf{S}^{1}}$ is a quasiconformal mapping. Moreover, $\Phi\left(\left.\widetilde{E}\right|_{\mathbf{S}^{1}}\right)$ and $\widetilde{E}$ induce the same group isomorphism between $G$ and $G^{\prime}$. This is due to the fact that the Douady-Earle extension of a homeomorphism of the unit circle is conformally natural; i.e.,

$$
\Phi\left(T_{1} \circ f \circ T_{2}\right)=T_{1} \circ \Phi(f) \circ T_{2}
$$

for any homeomorphism $f$ of the unit circle and any two isometries $T_{1}$ and $T_{2}$ of $\mathbf{D}$ (see [2]). Then $\Phi\left(\left.\widetilde{E}\right|_{\mathbf{S}^{1}}\right)$ projects to a quasiconformal homeomorphism

$$
\Phi_{\beta, \lambda}: S \rightarrow S^{\prime}
$$

which we call the Douady-Earle representation of the left earthquake map $E_{\beta, \lambda}: S \rightarrow$ $S^{\prime}$.

Lemma 1. Let $g_{1}, g_{2}$ be two hyperbolic transformations of the unit disk $\mathbf{D}$ with axes $L_{1}, L_{2}$ and translation lengths $\tau\left(g_{1}\right), \tau\left(g_{2}\right)$ respectively. If $L_{1}$ intersects $L_{2}$ at one point in $\mathbf{D}$, then $g_{3}=g_{1} \circ g_{2}$ is hyperbolic. Moreover, the translation length $\tau\left(g_{3}\right)$ of $g_{3}$ satisfies

$$
\tau\left(g_{2}\right)-\tau\left(g_{1}\right) \leq \tau\left(g_{3}\right) \leq \tau\left(g_{2}\right)+\tau\left(g_{1}\right)
$$


Proof. We label the repelling fixed point of $g_{1}$ by $a$ and the attracting fixed point by $c$, the repelling fixed point of $g_{2}$ by $b$ and the attracting fixed point by $d$. Then $L_{1}$ connects $a$ to $c$ and $L_{2}$ connects $b$ to $d$. Let $I$ be the circular arc between $c$ and $d$ not containing $a$ and $b$, and let $J$ be the circular arc between $a$ and $b$ not containing $c$ and $d$. See Figure 1 for an illustration. By studying the images of the endpoints of $I$ or $J$ under $g_{2}$ and then $g_{1}$, one can show that the intervals $I$ and $J$ satisfy $\left(g_{1} \circ g_{2}\right)(I) \subset I$ and $J \subset\left(g_{1} \circ g_{2}\right)(J)$. Therefore, $g_{3}=g_{1} \circ g_{2}$ has two fixed points, one in $I$ and another one in $J$. Then $g_{3}$ must be a hyperbolic transformation with axis joining the intervals $I$ and $J$.

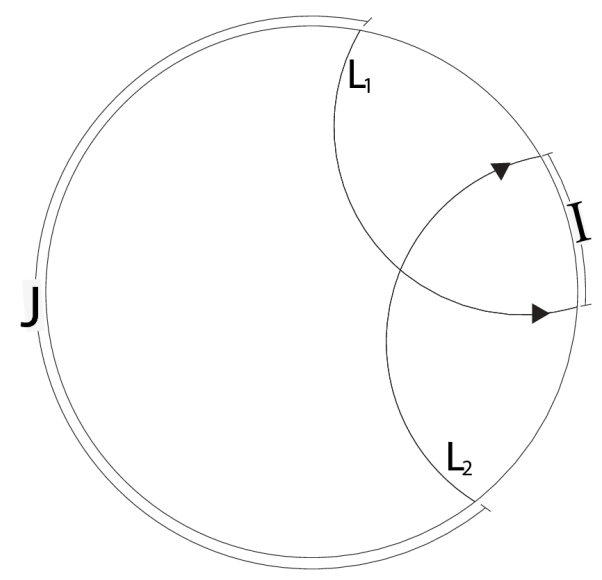

Figure 1. A reference figure for the proof of Lemma 1.

Let $p \in L_{1} \cap L_{2}$. Then $g_{2}^{-1}(p) \in L_{2}$ and

$$
\rho\left(g_{2}^{-1}(p), g_{1}(p)\right) \leq \rho\left(g_{2}^{-1}(p), p\right)+\rho\left(p, g_{1}(p)\right)=\tau\left(g_{2}\right)+\tau\left(g_{1}\right),
$$

where $\rho(\cdot, \cdot)$ denotes the hyperbolic metric on $\mathbf{D}$. Since

$$
\tau\left(g_{3}\right)=\inf _{z \in \mathbf{D}} \rho\left(z, g_{3}(z)\right) \leq \rho\left(g_{2}^{-1}(p), g_{1}(p)\right),
$$

it follows that

$$
\tau\left(g_{3}\right) \leq \tau\left(g_{2}\right)+\tau\left(g_{1}\right) .
$$

Applying a similar argument to $g_{2}=g_{3} \circ g_{1}^{-1}$, we obtain

$$
\tau\left(g_{2}\right) \leq \tau\left(g_{3}\right)+\tau\left(g_{1}^{-1}\right)=\tau\left(g_{3}\right)+\tau\left(g_{1}\right) .
$$

Thus

$$
\tau\left(g_{2}\right)-\tau\left(g_{1}\right) \leq \tau\left(g_{3}\right)
$$

Theorem 9. Let $\beta$ be a simple closed geodesic on $S$, and let

$$
\Phi_{\beta, \lambda}: S \rightarrow S^{\prime}
$$

be the Douady-Earle representation of a left earthquake map $E_{\beta, \lambda}: S \rightarrow S^{\prime}$ of shear $\lambda$ along $\beta$. Then for any $\gamma \in \Sigma_{S}$,

$$
l_{S}(\gamma)-i(\gamma, \beta) \lambda \leq l_{S^{\prime}}\left(\Phi_{\beta, \lambda}(\gamma)\right) \leq l_{S}(\gamma)+i(\gamma, \beta) \lambda
$$

where $i(\gamma, \beta)$ denotes the intersection number between $\gamma$ and $\beta$.

Proof. Let $\pi: \mathbf{D} \rightarrow S$ be a universal covering map and let $G$ be the Fuchsian group uniformizing $S$. Recall that $\left(\sigma_{\lambda}, \mathcal{L}\right)$ is a measured lamination with $\mathcal{L}$ consisting of all geodesics in $\pi^{-1}(\beta)$ and with the weight of each leaf equal to $\lambda$. Let $\widetilde{E}: \mathbf{D} \rightarrow \mathbf{D}$ is a left earthquake on $\mathbf{D}$ inducing $\left(\sigma_{\lambda}, \mathcal{L}\right)$. Then $S^{\prime}$ is uniformized by $G^{\prime}=\widetilde{E} G \widetilde{E}^{-1}$. 
Let $g \in G$ such that its axis $L$ projects to $\gamma$. Notice that $l_{S}(\gamma)$ and $l_{S^{\prime}}\left(\Phi_{\beta, \lambda}(\gamma)\right)$ are equal to the translation lengths of $g$ and $g^{\prime}=\widetilde{E} g \widetilde{E}^{-1}$, respectively. Let us assume that $\gamma \neq \beta$ and $i(\gamma, \beta)=0$. Then $L$ intersects none of the geodesics in the lamination $\mathcal{L}=\pi^{-1}(\beta)$. Without loss of generality, we may assume that $\left.\widetilde{E}\right|_{L}=$ id. Thus, for any point $z \in L, g(z) \in L$ and hence $g^{\prime}(z)=\left.\left.\widetilde{E}\right|_{A} g \widetilde{E}\right|_{A} ^{-1}(z)=g(z)$. By the identity principle, $g^{\prime} \equiv g$ and then they have the same translation length. It follows that

$$
l_{S}(\gamma)=l_{S^{\prime}}\left(\Phi_{\beta, \lambda}(\gamma)\right) \text { if } \gamma \neq \beta \text { and } i(\gamma, \beta)=0
$$

If $\gamma=\beta$, then a similar argument shows that the equation (3.2) holds.

Assume now that $\gamma \neq \beta$ and $i(\gamma, \beta)=n>0$. Let $p$ be a point in $\mathbf{D}$ which projects to a point in the intersection of $\gamma$ and $\beta$. Since $p$ and $g(p)$ project to the same point, the geodesic segment $[p, g(p)]$ crosses exactly $n+1$ lines in the lamination $\mathcal{L}$, denoted by $L_{1}, \cdots, L_{n+1}$, and $n$ gaps, denoted by $A_{1}, \cdots, A_{n}$. Without loss of generality, we may assume $\left.\widetilde{E}\right|_{A_{0}}=\mathrm{id}$, where $A_{0}$ is the gap that is separated from $A_{1}$ by $L_{1}$. Then

$$
\left.\widetilde{E}\right|_{A_{n}}=T_{1} \circ T_{2} \circ \ldots \circ T_{n},
$$

where $T_{k}$ is the hyperbolic transformation with axis $L_{k}$ and translation length $\lambda$. Notice that $g$ maps an open neighborhood $V$ contained in $A_{0}$ to a neighborhood contained in $A_{n}$. Thus,

$$
\left.g^{\prime}\right|_{V}=\left.\left(\widetilde{E} g \widetilde{E}^{-1}\right)\right|_{V}=\left.\left(T_{1} \circ \ldots T_{n-1} \circ T_{n} \circ g\right)\right|_{V} .
$$

By the identity principle,

$$
g^{\prime}=T_{1} \circ \ldots T_{n-1} \circ T_{n} \circ g
$$

on D. It remains to show that the translation lengths $\tau\left(g^{\prime}\right)$ and $\tau(g)$ of $g^{\prime}$ and $g$ satisfy

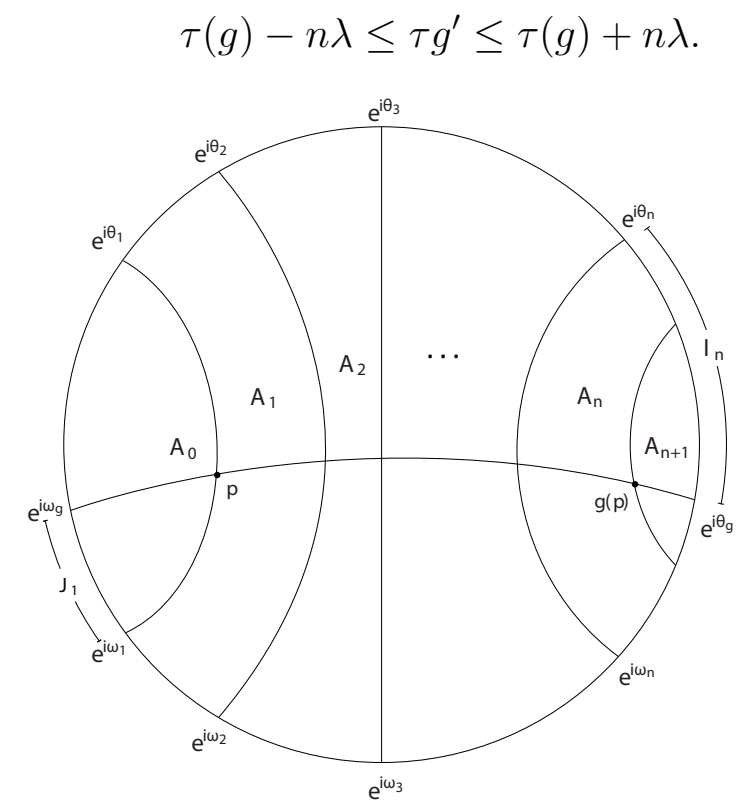

Figure 2. Illustration of a step in the proof of Theorem 9.

Clearly, the axis of $g$ intersects $L_{n}$. By Lemma 1, we obtain

$$
\tau(g)-\tau\left(T_{n}\right) \leq \tau\left(T_{n} \circ g\right) \leq \tau(g)+\tau\left(T_{n}\right) .
$$

Now we denote the attracting and repelling fixed points of $g$ by $e^{i \theta_{g}}$ and $e^{i \omega_{g}}$ and the ones of $T_{k}$ by $e^{i \theta_{k}}$ and $e^{i \omega_{k}}, k=1, \cdots, n+1$. Let $I_{k}$ be the circular arc on $\mathbf{S}^{1}$ between 
$e^{i \theta_{g}}$ and $e^{i \theta_{k}}$ not containing $e^{i \omega_{g}}$ and $e^{i \omega_{k}}, k=2,3, \cdots, n$, and let $J_{1}$ be the one on $\mathbf{S}^{1}$ between $e^{i \omega_{g}}$ and $e^{i \omega_{1}}$ not containing $e^{i \theta_{g}}$ and $e^{i \theta_{1}}$ (See Figure 2). Note that $g$ maps $L_{1}$ to $L_{n+1}$. In particular, it maps $e^{i \omega_{1}}$ to $e^{i \omega_{n+1}}$. It follows that $T_{n}$ maps $g\left(e^{i \omega_{1}}\right)=e^{i \omega_{n+1}}$ to a point closer to $e^{i \theta_{n}}$ than $g\left(e^{i \omega_{1}}\right)$. On the other hand, $g$ fixes $e^{i \omega_{g}}$ and then $T_{n}$ maps it to a point on the circular arc between $e^{i w_{g}}$ and $e^{i \theta_{n}}$ not containing $e^{i \omega_{1}}$. We conclude that $\left(T_{n} \circ g\right)\left(J_{1}\right) \supset J_{1}$. A similar argument shows $\left(T_{n} \circ g\right)\left(I_{n}\right) \subset I_{n}$. These imply that $T_{n} \circ g$ has a fixed point in $I_{n}$ and another one in $J_{1}$. It follows that the axis of $T_{n} \circ g$ must cross the axis of $T_{n-1}$. Using Lemma 1 again, we obtain

$$
\tau\left(T_{n} \circ g\right)-\tau\left(T_{n-1}\right) \leq \tau\left(T_{n-1} \circ T_{n} \circ g\right) \leq \tau\left(T_{n-1}\right)+\tau\left(T_{n} \circ g\right) .
$$

Similarly, we can see that $T_{n-1} \circ T_{n} \circ g\left(I_{n-1}\right) \subset I_{n-1}$ and $T_{n-1} \circ T_{n} \circ g\left(J_{1}\right) \supset J_{1}$. Hence $T_{n-1} \circ T_{n} \circ g$ has a fixed point in $I_{n-1}$ and another one in $J_{1}$. Then the axis of $T_{n-1} \circ T_{n} \circ g$ crosses the one of $T_{n-2}$. Lemma 1 can be applied again. Therefore, inductively, we are able to obtain

$$
\tau(g)-n \lambda=\tau(g)-\sum_{k=1}^{n} \tau\left(T_{k}\right) \leq \tau\left(g^{\prime}\right) \leq \tau(g)+\sum_{k=1}^{n} \tau\left(T_{k}\right)=\tau(g)+n \lambda .
$$

This means

$$
l_{S}(\gamma)-i(\gamma, \beta) \lambda \leq l_{S^{\prime}}\left(\Phi_{\beta, \lambda}(\gamma)\right) \leq l_{S}(\gamma)+i(\gamma, \beta) \lambda
$$

Combining the equations (3.2) and (3.3), we complete the proof.

Remark 3. Our approach to prove the previous theorem is analytic. Thanks to one of the referees for providing the following geometric approach. Let us use the same notation introduced in the previous proof, but let $p$ be a point on the hyperbolic plane projecting to a point on $\gamma$ not equal to any intersection point between $\gamma$ and $\beta$. Then the geodesic arc $[p, g(p)]$ crosses exactly $n$ geodesic lines $L_{k}, k=1,2, \cdots, n$, which are lifts of $\beta$. Let $a_{k}=[p, g(p)] \cap L_{k}$ for $k=1,2, \cdots, n$ and set $a_{0}=p$ and $a_{n+1}=g(p)$. The image $\tilde{E}([p, g(p)])$ is a union of geodesic $\operatorname{arcs}\left\{\tilde{E}\left(\left[a_{k}, a_{k+1}\right]\right)\right\}_{k=0}^{n}$ in the hyperbolic plane. Set $b_{k}^{-}=\tilde{E}\left(\left[a_{k-1}, a_{k}\right]\right) \cap \tilde{E}\left(L_{k}\right)$ and $b_{k}^{+}=\tilde{E}\left(\left[a_{k}, a_{k+1}\right]\right) \cap \tilde{E}\left(L_{k}\right)$ for $k=1,2, \cdots, n$, where $\tilde{E}$ also represents the extension to the hyperbolic plane of the part of $\tilde{E}$ defined on the gap containing $\left(a_{k-1}, a_{k}\right)$ for each $k=1,2, \cdots, n+1$. Then, by definition, the geodesic arc $\left[b_{k}^{-}, b_{k}^{+}\right]$lies on the line $\tilde{E}\left(L_{k}\right)$ and has length $\lambda$ (Lemma 3.6 of [8]). Therefore, the concatenation

$$
\delta=\left(\bigcup_{k=1}^{n+1} \tilde{E}\left(\left[a_{k-1}, a_{k}\right]\right)\right) \cup\left(\bigcup_{k=1}^{n}\left[b_{k}^{-}, b_{k}^{+}\right]\right)
$$

has length $l_{S}(\beta)+n \lambda$. Because $\tilde{E} \circ g=g^{\prime} \circ \tilde{E}$, the piecewise geodesic path

$$
\bigcup_{k \in \mathbf{Z}}\left(g^{\prime}\right)^{k}(\tilde{E}(\delta))
$$

is a cross cut, invariant under the action of $g^{\prime}$ and represents a closed curve on $S^{\prime}$ corresponding to $\beta$ via markings. Thus, the length $l_{S^{\prime}}(\beta)$ is at most $l_{S}(\beta)+n \lambda$.

\section{Proof of main results}

Let $S$ be a Riemann surface with corresponding Fuchsian group $G$ and let $\gamma$ be an essential simple closed curve on $S$. A ring domain $R$ on $S$ is a set conformally equivalent to an annulus $\{z: a<|z|<1\}$ in the complex plane, where $a$ is a nonnegative real number uniquely determined by $R$. The conformal modulus of $R$ is defined 
by $\operatorname{Mod}(R)=(2 \pi)^{-1}|\log a|$. A ring domain $R$ is said to be of homotopy type $\gamma$ if there exists a simple closed curve $\gamma_{0} \subset R$ freely homotopic to $\gamma$ and separating the boundary components of $R$. If $\gamma$ is not homotopic to a puncture then any element $g \in G$ covering $\gamma$ is hyperbolic and $R_{S, \gamma}^{\prime}=\mathbf{H} /<g>$ is a ring domain that covers $S$. It is straightforward to check that $\operatorname{Mod}\left(R_{S, \gamma}^{\prime}\right)=\pi / l_{S}(\gamma)$. Moreover, any ring domain $R$ in $S$, of homotopy type $\gamma$, can be conformally embedded into $R_{S, \gamma}^{\prime}$ in such a way that both ring domains have the same homotopy type (see [20] or [14]). Then

$$
\operatorname{Mod}(R) \leq \operatorname{Mod}\left(R_{S, \gamma}^{\prime}\right)=\frac{\pi}{l_{S}(\gamma)}
$$

for any ring domain $R$ in $S$ of homotopy type $\gamma$.

Let $\varphi$ be a holomorphic quadratic differential on $S$. An arc $\gamma$ is a trajectory of $\varphi$ if it is a maximal horizontal arc, i.e., if $\arg \varphi(z) d z^{2}=0$ along $\gamma$. Every closed trajectory $\gamma_{0}$ of $\varphi$ is embedded in a unique maximal ring domain $R_{0}$ swept out by closed trajectories. Two ring domains $R_{0}$ and $R_{1}$ associated with $\gamma_{0}$ and $\gamma_{1}$ are disjoint or identical.

The quadratic differential $\varphi$ is said to be of closed trajectories if its non closed trajectories cover a set of measure zero. The associated ring domains of such $\varphi$ divide $S$ into ring domains swept out by closed trajectories. We say that $\varphi$ is simple if it has only one associated ring domain.

A set of homotopically non trivial simple closed curves on $S$ is called an admissible system of simple closed curves provided that they are mutually disjoint and belong to different homotopy classes. Strebel [20] proved the following theorem.

Theorem 10. (Strebel) Let $S$ be a Riemann surface and $\left\{\gamma_{1}, \cdots, \gamma_{n}\right\}$ an admissible system of simple closed curves on $S$ that are not homotopic to any puncture on $S$. Then for arbitrary positive numbers $b_{k}, k=1,2, \cdots, n$, there exists a holomorphic quadratic differential $\varphi$ on $S$ with closed trajectories such that its associated ring domains $R_{k}$ are of homotopy types $\gamma_{k}$ and heights $b_{k}$ respectively for $k=1,2, \cdots, n$. Furthermore, the quadratic differential $\varphi$ is uniquely determined and has a finite norm

$$
\|\varphi\|=\sum_{k=1}^{n} b_{k}^{2} / M_{k},
$$

where $M_{k}$ is the modulus of $R_{k}, k=1, \cdots, n$.

For each simple closed curve $\beta \in \Sigma_{S}$, let $\varphi_{\beta}$ be the unique simple quadratic differential with

$$
\left\|\varphi_{\beta}\right\|=\iint_{S}\left|\varphi_{\beta}\right| d x d y=1
$$

such that its closed trajectories are freely homotopic to $\beta$. We will denote the associated ring domain of $\varphi_{\beta}$ by $R_{S, \beta}$. Notice that (4.1) implies

$$
\operatorname{Mod}\left(R_{S, \beta}\right) \leq \operatorname{Mod}\left(R_{S, \beta}^{\prime}\right)=\frac{\pi}{l_{S}(\beta)}
$$

Let $S_{0}$ be a hyperbolic Riemann surface that is not an annulus. Let $\beta$ be a simple closed geodesic on $S_{0}$ and $\varphi_{\beta}$ be the corresponding simple quadratic differential. For each $t \in(0,1), \mu_{t}=-t \frac{\bar{\varphi}_{\beta}}{\left|\varphi_{\beta}\right|}$ defines a Beltrami differential on $S_{0}$. We use $\mu_{t}$ to give a new conformal structure on $S_{0}$.

For any local parameter $z: U \rightarrow V$ on $S_{0}$, let $z^{\prime}: V \rightarrow z^{\prime}(V)$ be a quasiconformal mapping with complex dilatation $\left.\mu_{t}\right|_{V}$. Since $\mu_{t}$ is a Beltrami differential, the new parameters $z^{\prime}$ give a new complex structure on $S_{0}$. We will denote the new Riemann 
surface by $S_{t}$. The identity map id $\operatorname{id}_{t} S_{0} \rightarrow S_{t}$ is a Teichmüller mapping with initial quadratic differential $\varphi_{\beta}$ and some terminal quadratic differential $\psi_{t}$. The mapping $\mathrm{id}_{t}$ can be expressed as

$$
\operatorname{id}_{t}: \zeta \mapsto \zeta^{\prime}=\frac{\zeta-t \bar{\zeta}}{1-t}
$$

where $\zeta$ and $\zeta^{\prime}$ are natural parameters of $\varphi_{\beta}$ and $\psi_{t}$ respectively. It follows that $i d_{t}$ stretches the vertical trajectories of $\varphi_{\beta}$ by a factor $K_{t}=\frac{1+t}{1-t}$ and leaves the lengths of the horizontal trajectories invariant. Then the quadratic differential $\psi_{t}$ is also simple and its associated ring domain $R_{S_{t}, \beta}$ coincides with $R_{S_{0}, \beta}$. Nonetheless, the conformal moduli are different; in fact it is easily seen that

$$
\operatorname{Mod}\left(R_{S_{t}, \beta}\right)=K_{t} \operatorname{Mod}\left(R_{S_{0}, \beta}\right) .
$$

Let $\beta_{t}$ be the geodesic on $S_{t}$ homotopic to $\beta$, it follows from the above equality and (4.2) that

$$
l_{S_{t}}\left(\beta_{t}\right) \leq \frac{\pi}{\operatorname{Mod}\left(R_{S_{t}, \beta}\right)}=\frac{\pi}{K_{t} \operatorname{Mod}\left(R_{S_{0}, \beta}\right)}
$$

It follows that $l_{S_{t}}\left(\beta_{t}\right) \rightarrow 0$ as $t \rightarrow 1$.

Consider the geodesic ray (in the Teichmüller metric) given by

$$
\alpha(t)=\left[S_{t}, \mathrm{id}_{t}\right], \quad t \in[0,1),
$$

and another continuous path defined by

$$
\alpha^{*}(t)=\left[S_{t}^{\prime}, \Phi_{\beta_{t}, \lambda_{t}} \circ \mathrm{id}_{t}\right], \quad t \in[0,1),
$$

where $\lambda_{t}$ is a positive parameter continuously depending on $t$ and $\Phi_{\beta_{t}, \lambda_{t}}$ is the Douady-Earle representation of the left earthquake $E_{\beta_{t}, \lambda_{t}}$ of twist $\lambda_{t}$ along $\beta_{t}$. We show the following theorem.

Theorem 11. Let $\alpha(t)$ and $\alpha^{*}(t)$ be as above. If $\lambda_{t}=o\left(\left|\log l_{S_{t}}\left(\beta_{t}\right)\right|\right)$ as $t \rightarrow 1$, then

$$
\lim _{t \rightarrow 1} d_{L}\left(\alpha(t), \alpha^{*}(t)\right)=0 .
$$

Proof. Suppose $\gamma$ is a closed geodesic in $S_{t}$. Then Theorem 9 implies

$$
l_{S_{t}^{\prime}}\left(\Phi_{\beta_{t}, \lambda_{t}}(\gamma)\right)=l_{S_{t}}(\gamma) \text { if } i\left(\gamma, \beta_{t}\right)=0 .
$$

On the other hand, if $i\left(\gamma, \beta_{t}\right)>0$, then Theorem 9 implies

$$
1-\frac{i\left(\gamma, \beta_{t}\right) o\left(\left|\log l_{S_{t}}\left(\beta_{t}\right)\right|\right)}{l_{S_{t}}(\gamma)} \leq \frac{l_{S_{t}^{\prime}}\left(\Phi_{\beta_{t}, \lambda_{t}}(\gamma)\right)}{l_{S_{t}}(\gamma)} \leq 1+\frac{i\left(\gamma, \beta_{t}\right) o\left(\left|\log l_{S_{t}}\left(\beta_{t}\right)\right|\right)}{l_{S_{t}}(\gamma)}
$$

By inequality (4.3), $l_{S_{t}}\left(\beta_{t}\right)$ is small when $t$ is close to 1 . In this situation, we know, by the Collar Lemma, that $\beta_{t}$ has a collar neighborhood of approximate width $D_{t} \approx \log \left(16 / l_{S_{t}}\left(\beta_{t}\right)\right)$. Then

$$
l_{S_{t}}(\gamma) \geq i(\gamma, \beta) \log \left(16 / l_{S_{t}}\left(\beta_{t}\right)\right) .
$$

Thus, if $t$ is close to 1 and $i(\gamma, \beta)>0$, then

$$
1-\frac{o\left(\left|\log l_{S_{t}}\left(\beta_{t}\right)\right|\right)}{\log \frac{16}{l_{S_{t}}\left(\beta_{t}\right)}} \leq \frac{l_{S_{t}^{\prime}}\left(\Phi_{\beta_{t}, \lambda_{t}}(\gamma)\right)}{l_{S_{t}}(\gamma)} \leq 1+\frac{o\left(\left|\log l_{S_{t}}\left(\beta_{t}\right)\right|\right)}{\log \frac{16}{l_{S_{t}}\left(\beta_{t}\right)}}
$$

By using (4.5) if $i\left(\gamma, \beta_{t}\right)=0$ or (4.6) if $i\left(\gamma, \beta_{t}\right)>0$ and letting $t \rightarrow 1$, we obtain

$$
\lim _{t \rightarrow 1} d_{L}\left(\alpha(t), \alpha^{*}(t)\right)=0 \text {. }
$$


Remark 4. As a Teichmüller geodesic, the curve $\alpha(t)$ leaves any compact subset of $T\left(S_{0}\right)$ under the metric $d_{T}$ as $t \rightarrow 1$. It also leaves any compact subset under the metric $d_{L}$ since $l_{S_{t}}\left(\beta_{t}\right) \rightarrow 0$ as $t \rightarrow 1$; that is,

$$
d_{L}(\alpha(t), \alpha(0)) \rightarrow \infty \text { as } t \rightarrow 1 .
$$

Using the previous theorem, we obtain

$$
d_{L}\left(\alpha^{*}(t), \alpha(0)\right) \rightarrow \infty \text { as } t \rightarrow 1
$$

Then the inequality (1.3) implies

$$
d_{T}\left(\alpha^{*}(t), \alpha(0)\right) \rightarrow \infty \text { as } t \rightarrow 1
$$

Therefore, the curve $\alpha^{*}(t)$ leaves any compact subset of $T\left(S_{0}\right)$ under both $d_{T}$ and $d_{L}$ metrics as well.

Theorem 12. Let $\alpha(t)$ and $\alpha^{*}(t)$ be as above.

(1) If $\lambda_{t} \rightarrow \infty$ as $t \rightarrow 1$, then $\lim _{t \rightarrow 1} d_{T}\left(\alpha(t), \alpha^{*}(t)\right)=\infty$.

(2) If there exist two positive constants $C_{1}<C_{2}$ such that $C_{1} \leq \lambda_{t} \leq C_{2}$ for all $t$, then there exist two positive constants $m<M$ such that $m \leq$ $d_{T}\left(\alpha(t), \alpha^{*}(t)\right) \leq M$ for all $t$.

Proof. Let $\pi_{t}: \mathbf{D} \rightarrow S_{t}$ be the universal covering map and let $G_{t}$ be the group uniformizing $S_{t}$. Denote by $\widetilde{E}_{t}: \mathbf{D} \rightarrow \mathbf{D}$ the left earthquake corresponding to $E_{\beta_{t}, \lambda_{t}}$. By inequality (3.1) and Theorem 6, we obtain

$$
\left\|\widetilde{E}_{t}\right\|_{\mathrm{cr}} \geq \frac{1}{C^{\prime}}\left\|\sigma_{\lambda_{t}}\right\|_{\mathrm{Th}} \geq \frac{1}{C^{\prime}} \lambda_{t} .
$$

If $\lambda_{t} \rightarrow \infty$ as $t \rightarrow 1$, then the cross-ratio distortion norm approaches $\infty$ as $t \rightarrow 1$. It follows from Theorem 8 that the maximal dilatation of any extension of $\widetilde{E}_{t}$ is approaching $\infty$ as $t \rightarrow 1$. Since any lift $F_{t}$ of the extremal quasiconformal mapping in the homotopy class of $\Phi_{\beta_{t}, \lambda_{t}}$ satisfies $\left.F_{t}\right|_{\mathbf{R}}=\left.\left(T \circ \widetilde{E}_{t}\right)\right|_{\mathbf{R}}$ for some Möbius transformation $T$, it follows that $K\left(F_{t}\right) \rightarrow \infty$ as $t \rightarrow 1$. That is,

$$
\lim _{t \rightarrow 1} d_{T}\left(\alpha(t), \alpha^{*}(t)\right)=\infty
$$

and the first part of the theorem follows.

Now suppose that $\lambda_{t} \leq C$ for all $t$. By inequality (3.1) and Theorem 6, we now obtain

$$
C^{\prime} \max \left\{C_{2}, 2 C_{2} / D_{t}\right\} \geq C^{\prime}\left\|\sigma_{\lambda_{t}}\right\|_{\mathrm{Th}} \geq\left\|\widetilde{E}_{t}\right\|_{\mathrm{cr}} \geq \frac{1}{C^{\prime}}\left\|\sigma_{\lambda_{t}}\right\|_{\mathrm{Th}} \geq \frac{C_{1}}{C^{\prime}}>0
$$

where as before, $D_{t} \approx \log \left(16 / l_{S_{t}}\left(\beta_{t}\right)\right)$ is the width of a collar neighborhood around the geodesic $\beta_{t}$ and $\widetilde{E}_{t}: \mathbf{D} \rightarrow \mathbf{D}$ is a left earthquake map corresponding to $E_{\beta_{t}, \lambda_{t}}$. Since the cross-ratio distortion norm of $\widetilde{E}_{t}$ is bounded from above, it follows from Theorem 7 that there exists a constant $M$ such that $K_{e}\left(\widetilde{E}_{t}\right) \leq e^{M}$ for all $t$. In particular

$$
d_{T}\left(\alpha(t), \alpha^{*}(t)\right) \leq M \text { for all } t .
$$

On the other hand, since the cross-ratio distortion norm of $\widetilde{E}_{t}$ is bounded from below, we know, by Proposition 1 , that there exists a constant $m>0$ such that $e^{m} \leq K_{e}\left(\widetilde{E}_{t}\right)$ for all $t$. Thus,

$$
m \leq d_{T}\left(\alpha(t), \alpha^{*}(t)\right) \text { for all } t .
$$


From Theorem 4 in [19], we know that the curve $\alpha(t), t \in[0,1)$, is a Teichmüller geodesic in $T\left(S_{0}\right)$ emanating from the base point. Now let $\lambda_{t}$ be a nonnegative parameter continuously depending on $t \in[0,1)$ and satisfying that $\lambda_{t}=o\left(\mid \log \left(l_{S_{t}}\left(\beta_{t}\right) \mid\right)\right.$ and $\lambda_{t} \rightarrow \infty$ as $t \rightarrow 1$. Then we obtain the first part of Theorem 1 by applying Theorem 11 and the first part of Theorem 12 to the curve $\alpha^{*}(t)$ given in (4.4). On the other hand, by letting $\lambda_{t}$ be bounded from above and from below by positive constants, we obtain the second part of Theorem 1 by using Theorem 11 and the second part of Theorem 12 .

\section{References}

[1] Abikoff, W.: The real analytic theory of Teichmüller space. - Lecture Notes in Math. 820, Springer-Verlag, Berlin-Heidelberg-New York, 1980.

[2] Doundy, A., and C. J. EARle: Conformally natural extension of homeomorphims of the circle. - Acta Math. 157, 1986, 23-48.

[3] Gardiner, F. P., J. Hu, and N. LAKIC: Earthquake curves. - Contemp. Math. 311, 2002, $141-195$.

[4] Hu, J.: Earthquake measure and cross-ratio distortion. - Contemp. Math. 355, 2004, 285-308.

[5] Hu, J.: Earthquakes on the hyperbolic plane. - In: Handbook of Teichmüller theory III (edited by A. Papadopoulos), IRMA Lect. Math. Theor. Phys. 17, Eur. Math. Soc., 2012, 71-122.

[6] Hu, J., and O. Muzician: Cross-ratio distortion and Douady-Earle extension: I. A new upper bound on quasiconformality. - J. London Math. Soc. 86:2, 2012, 387-406.

[7] KeEN, L.: Collars on Riemann surfaces. - In: Discontinuous groups and Riemann surfaces, Proceedings of the Maryland Conf. 1973, Ann. of Math. Stud. 79, 1974, 263-268.

[8] Kerckhoff, S.: The Nielsen realization problem. - Ann. of Math. (2) 177, 1983, 235-265.

[9] Lehto, O., and K. I. Virtanen: Quasiconformal mapping. - Springer, New York, 1973.

[10] LI, Z.: Teichmüller metric and length spectrum of Riemann surface. - Scientia Sinica Ser. A $24,1986,265-274$.

[11] Li, Z.: Length spectrum of Riemann surfaces and Teichmüller metric. - Bull. London Math. Soc. 35, 2003, 247-254.

[12] LiU, L.: On the length spectrums of non-compact Riemann surfaces. - Ann. Acad. Sci. Fenn. Math. 24, 1999, 11-22.

[13] Liu, L., Z. Sun, and H. Wei: Topological equivalence of metrics in Teichmüller space. - Ann. Acad. Sci. Fenn. Math. 33, 2008, 159-170.

[14] Maskit, B.: Comparison of hyperbolic and extremal lengths. - Ann. Acad. Sci. Fenn. Math. Ser. A I Math. 10, 1985, 381-386.

[15] S̆ARIĆ, D.: Real and complex earthquakes. - Trans. Amer. Math. Soc. 358, 2006, 233-249.

[16] ShigA, H.: On a distance defined by the length spectrum on Teichmüller space. - Ann. Acad. Sci. Fenn. Math. 28, 2003, 315-326.

[17] Sorvali, T.: The boundary mapping induced by an isomorphism of covering groups. - Ann. Acad. Sci. Fenn. Ser. A I Math. 526, 1972, 1-31.

[18] Sorvali, T.: On Teichmüller spaces of tori. - Ann. Acad. Sci. Fenn. Ser. A I Math. 1, 1975, $7-11$.

[19] Strebel, K.: On quasiconformal mappings of open Riemann surfaces. - Comment. Math. Helv. 53, 1978, 301-321.

[20] Strebel, K.: Quadratic differentials. - Springer-Verlag, Berlin-Heidelberg-New York-Tokyo, 1984.

[21] Thurston, W.: Earthquakes in two-dimensional hyperbolic geometry. - In: Low-dimensional topology and Kleinian groups 122, London Math. Soc., 1986, 91-112.

Received 9 March 2014 • Revised received 26 March 2015 • Accepted 12 August 2015 